\title{
ESTUDIO DE LA CARCASA DE ALPACAS (Vicugna pacos) EN RELACIÓN AL PESO Y CLASIFICACIÓN CÁRNICA
}

\author{
Carcass Study in Relation to Weight and Grading Meat in Alpacas \\ (VICUGNA PACOS)
Edgar Quispe P. ${ }^{1,4}$, Adolfo Poma G. ${ }^{1}$, Omar Siguas R. ${ }^{1}, M^{\mathrm{a}}$ José Berain A. ${ }^{2}$, Antonio Purroy U. ${ }^{3}$

\section{RESUMEN}

El presente trabajo se realizó en el Camal Municipal de Huancavelica, Perú, ubicado a $3680 \mathrm{msnm}$. Se tomaron datos de 200 alpacas Huacaya con el objetivo de caracterizar las carcasas en función a peso vivo (PV), peso de la carcasa caliente (PCC), peso de carcasa fría (PCF) y grados de clasificación cárnica. Las medias de PV fueron de 49.5 y $55.6 \mathrm{~kg}$ para machos y hembras, y 51.3 y $53.1 \mathrm{~kg}$ para animales de 4 dientes y boca llena, respectivamente. Las medias de PCC fueron de 27.3 y $30.0 \mathrm{~kg}$ para machos y hembras, y $28.1 \mathrm{y}$ $28.8 \mathrm{~kg}$ para animales de 4 dientes y boca llena, respectivamente. Las medias de PCF fueron de 26.9 y $29.6 \mathrm{~kg}$ para machos y hembras, y de 27.7 y $28.4 \mathrm{~kg}$ para alpacas de 4 dientes y boca llena, respectivamente. La mayor parte de las carcasas correspondieron a clasificaciones de segundo grado y tipo industrial (38.0 y $36.5 \%$, respectivamente), no encontrándose carcasas de tipo extra. Ecuaciones lineales simples para predecir el PCC y PCF a partir del PV resultaron con coeficientes de determinación del 68.5 y $68.3 \%$, respectivamente. Además, se encontraron correlaciones positivas y altamente significativas $(p<0.01)$ entre las variables en estudio con valores no menores a 0.8 , obteniéndose una relación casi perfecta entre PCC y PCF.

Palabras clave: alpaca, peso, carcasa, clasificación

\section{ABSTRACT}

This research was carried out in the Huancavelica slaughterhouse, located at 3680 meters above sea level. Data were collected from 200 Huacaya alpacas to characterize the carcasses in relation to body weight (BW), hot carcass weight (HCW), cold carcass weight $(\mathrm{CCW})$ and grading meat. BW was 49.5 and $55.6 \mathrm{~kg}$ for males and females, and 51.3 and $53.1 \mathrm{~kg}$ for 4-tooth and full mouth animals respectively. HCW were 27.3 and $30.0 \mathrm{~kg}$ for males and females, and 28.1 and $28.8 \mathrm{~kg}$ for 4-tooth and alpaca full mouth respectively. CCW were 26.9 and $29.6 \mathrm{~kg}$ for males and females, and 27.7 and $28.4 \mathrm{~kg}$ for 4 -tooth and

\footnotetext{
${ }^{1}$ Programa de Mejora de Camélidos Sudamericanos, Departamento de Zootecnia, Universidad Nacional de Huancavelica - UNA, Huancavelica, Perú

${ }^{2}$ Departamento de Ciencias del Medio Natural, ${ }^{3}$ Departamento de Producción Agraria, Universidad Pública de Navarra - UPNA, Pamplona, España

${ }^{4}$ E-mail: edgarquispe62@yahoo.com
} 
alpaca full mouth respectively. Most carcasses were classified as second grade (38.0\%) and industrial meat (36.5\%) and none was of extra grade. Simple linear equations to predict HCW and CCW from BW showed coefficients of determination of 68.5 and $68.3 \%$ respectively. Moreover, highly significant positive correlations $(p<0.01)$ were found between the variables under study with values over 0.8 , yielding an almost perfect match between $\mathrm{HCW}$ and $\mathrm{CCW}$.

Key words: alpaca, weight, carcass, grading

\section{INTRODUCCIÓN}

Las alpacas representan un recurso importante para la producción de carne en las familias de la zona alto andina (Fairfield, 2006), debido a restricciones de adaptabilidad del ovino y del bovino en zonas ganaderas sobre los $4000 \mathrm{msnm}$. Se requiere estudiar debidamente el potencial carnicero de la alpaca para mejorar los sistemas de crianza e incrementar los niveles de productividad, lo cual representa la mejor estrategia de lucha contra la pobreza en poblaciones sudamericanas que viven en zonas alto andinas (Wheeler, 1993). Se estima que en el Perú se benefician entre 0.5 a 1 millón de alpacas por año, con una producción de $11000 \mathrm{TM}$ de carne (Hack, 2001), con rendimientos y pesos de carcasa alrededor del $50 \%$ y $23 \mathrm{~kg}$, respectivamente (Bustinza et al., 1975).

En las comunidades campesinas, la venta de alpacas para carne la lleva a cabo el jefe de familia, pero quien selecciona los animales es la esposa, como responsable del manejo y conocedora del hato. Durante el año puede ejecutarse una saca normal y otra forzada. La normal se realiza al final de la época de lluvias (marzo a julio) coincidiendo con el inicio de la escasez de pastos y cuando los animales se encuentran en regular condición de carnes (Mamani, 1989). En estas sacas, las mejores alpacas se venden en pie $\mathrm{y}$ al mejor precio, y el resto se beneficia para ofrecerlos como carne fresca o para la elaboración de charqui. Las sacas forzadas se realizan en cualquier época del año como re- curso económico para solucionar las necesidades de la familia (UNECE, 2006).

Lamentablemente, el sistema de crianza de la alpaca no está debidamente tecnificado, quedando muchos vacíos por conocer y mejorar, de allí que se tenga una baja eficiencia reproductiva, alta tasa de mortalidad y, consecuentemente, reducida producción de carne en todas las unidades productivas de los países andinos (Flores, 1982; Bustinza et al., 1985). Por otro lado, la carne tiene buenos parámetros tecnológicos de calidad (Saadoun y Cabrera, 2008; Salvá et al., 2009), pero aún se tienen problemas de aceptación debido principalmente a prejuicios socio-culturales, condiciones sanitarias (principalmente por la presencia de Sarcocystis aucheniae) y abastecimiento (Fairfield, 2006).

Tapia (1965), trabajando con hembras adultas de descarte encontró un rendimiento de carcasa de 53.5\%, y Téllez (1988) trabajando con hembras y machos adultos de desecho y carcasas con 24 horas de oreo, encontró un rendimiento de $54.0 \%$. Asimismo, en el trabajo de Ampuero y Alarcón (1989), con machos de 18 meses, se encontraron rendimientos de $52 \%$. En base a estos resultados, se planteó el presente estudio para: caracterizar el peso vivo, peso de la canal caliente y fría, y grado de clasificación; construir una ecuación de regresión para predecir el peso de carcasa fría o peso de carcasa caliente en función al peso vivo; y evaluar el efecto del grado de clasificación sobre el peso vivo, peso de la canal caliente y peso de la canal fría. 


\section{Materiales y Métodos}

El estudio se realizó entre los meses de mayo a julio de 2006 en el Camal Municipal de Huancavelica, a una altitud de $3680 \mathrm{msnm}$, con una temperatura ambiental promedio de $11.5^{\circ} \mathrm{C}$. La infraestructura e instalaciones del establecimiento son precarias, y el proceso del beneficio que incluye desde la recepción del animal hasta el despacho de la carne no siempre cumple las adecuadas condiciones sanitarias y de manejo.

Se trabajó con 200 alpacas. Se tomaron datos de edad (4 dientes y boca llena) y sexo (hembra y macho). El peso vivo (PV) se registró en la zona de recepción, entre las 14:00 y 15:00 horas, previo al desuello, con un mínimo de cinco horas de ayuno y con vellón (se estima que el peso del vellón estaba entre 2 a $3 \mathrm{~kg}$ ). El peso de la canal se tomó en fresco (peso de canal caliente, PCC), entre las 15:00 y 16:00 horas, y luego del proceso de oreo (peso de canal fría, PCF), a las 12 horas aproximadamente. La clasificación de la carcasa se realizó en la sala de oreo, tomando en cuenta la edad, sexo, y la cubierta e infiltración de grasa, considerando los grados de carne extra, primera, segunda e industrial (RTC, 1995; INDECOPI, 2005).

Los datos fueron analizados utilizando el paquete estadístico Software SPSS. Se determinaron promedios, desviación estándar y coeficiente de variación para cada uno de los criterios en estudio. Para la evaluación de las relaciones entre las variables cuantitativas se determinaron las correlaciones de Pearson, y para determinar el efecto de la clasificación de la canal sobre las variables de peso vivo, PCC y PCF se utilizó el diseño de bloques completamente al azar con submuestreo, cuyo modelo es:

$$
\mathrm{Y}_{\mathrm{ij}}=\mu+\frac{1}{100_{\mathrm{i}}}+\beta_{\mathrm{j}}+\left(\frac{1}{100_{\mathrm{i}}} \beta_{\mathrm{j}}\right)+\mathrm{e}_{\mathrm{ij}}
$$

dónde $Y_{i j}$ es la i-ésima j-ésima observación; $\frac{1}{100_{i}}$ es el efecto de la i-ésima clasificación (Tratamiento); $\beta_{\mathrm{j}}$ es el efecto del i-ésimo sexo (Bloque); $\left(\frac{1}{100_{i}} \beta_{\mathrm{j}}\right)$ es el efecto del i-ésimo, j-ésimo error de muestreo; y $e_{i j}$ es el efecto del i-ésimo, j-ésimo error experimental.

Para la predicción del peso de la carcasa tanto fría como caliente en función al peso vivo se utilizó la regresión lineal, cuyo modelo es: $Y_{i j}=a+b_{i}\left(X_{i}\right)+e_{i j}$; donde: $Y_{i j}$ es la iésima j-ésima variable dependiente (Peso de canal frío o peso de canal caliente); $b_{i}$ es el coeficiente de regresión; $X_{i}$ es el valor de la i-ésima variable independiente (Peso vivo); y $e_{i j}$ es el efecto del i-ésimo, j-ésimo error experimental.

\section{RESUltados}

Los resultados obtenidos para $\mathrm{PV}, \mathrm{PCC}$ y PCF se muestran en el Cuadro 1. Los machos tuvieron pesos menores que las hembras $(\mathrm{p}<0.01)$ a la prueba de " $\mathrm{t}$ " de Student, mientras que en el factor edad, si bien los animales adultos mostraron pesos ligeramente mayores que los animales jóvenes, esta diferencia no fue estadísticamente significativa.

En la Fig. 1 se puede observar que no se encontró canales clasificadas con calidad extra, y que el $75 \%$ de las canales fueron clasificadas como carne de segunda e industrial, mientras que un porcentaje reducido se clasificó cómo carne de primera. Asimismo, se pudo observar que la mayor parte de las canales clasificadas como carne industrial se debió en gran medida a la presencia de Sarcocistes.

En el Cuadro 2 se muestra una correlación altamente significativa entre $\mathrm{PV}$ con PCC y PV con PCF ( $r=0.83$ y r $=0.83$, respectivamente). Asimismo, la correlación entre PCF y PCC (0.99) fue altamente significativa, con un valor positivo casi perfecto. Las correlaciones del rendimiento de la carcasa con PCC y PCF son positivas pero bajas, mientras que con el PV resultó ser negativo y también baja; sin embargo, altamente significativas en todos los casos. 
Cuadro 1. Peso vivo y de la canal $(\mathrm{kg})$ de alpacas adultas sacrificadas en el camal de Huancavelica, Perú, según sexo y edad

\begin{tabular}{|c|c|c|c|c|c|c|c|}
\hline \multirow{2}{*}{ Variable } & \multirow{2}{*}{$\begin{array}{c}\mathrm{N}^{\circ} \mathrm{de} \\
\text { animales }\end{array}$} & \multicolumn{2}{|c|}{ Peso vivo } & \multicolumn{2}{|c|}{ Peso canal caliente } & \multicolumn{2}{|c|}{ Peso canal fría } \\
\hline & & Media & I.C. ${ }^{1}$ & Media & I.C. & Media & I.C. \\
\hline \multicolumn{8}{|l|}{ Sexo } \\
\hline - Madho & 83 & $49.5^{2}$ & $47.8 \pm 51.1$ & $27.3^{2}$ & $26.4 \pm 28.2$ & $26.9^{2}$ & $26.0 \pm 27.7$ \\
\hline - Hembra & 117 & $55.6^{\mathrm{b}}$ & $54.1 \pm 57.1$ & $30.0^{\mathrm{b}}$ & $29.0 \pm 31.0$ & $29.6^{\mathrm{b}}$ & $28.6 \pm 30.5$ \\
\hline \multicolumn{8}{|l|}{ Edad } \\
\hline-4 dientes & 87 & $51.3^{2}$ & $49.7 \pm 52.9$ & $28.1^{2}$ & $27.2 \pm 29.1$ & $27.7^{2}$ & $26.8 \pm 28.6$ \\
\hline - Boca llena & 113 & $53.1^{2}$ & $51.3 \pm 55.0$ & $28.8^{2}$ & $27.8 \pm 29.9$ & $28.4^{2}$ & $27.4 \pm 29.4$ \\
\hline
\end{tabular}

1 Irtervalo de c onfiarza del $95 \%$

${ }^{\mathrm{a}}$ Superíndices diferentes entre las variables sexo y edady dentro de columnas indican diferencias estadísticas al nivel de $p<0.01$

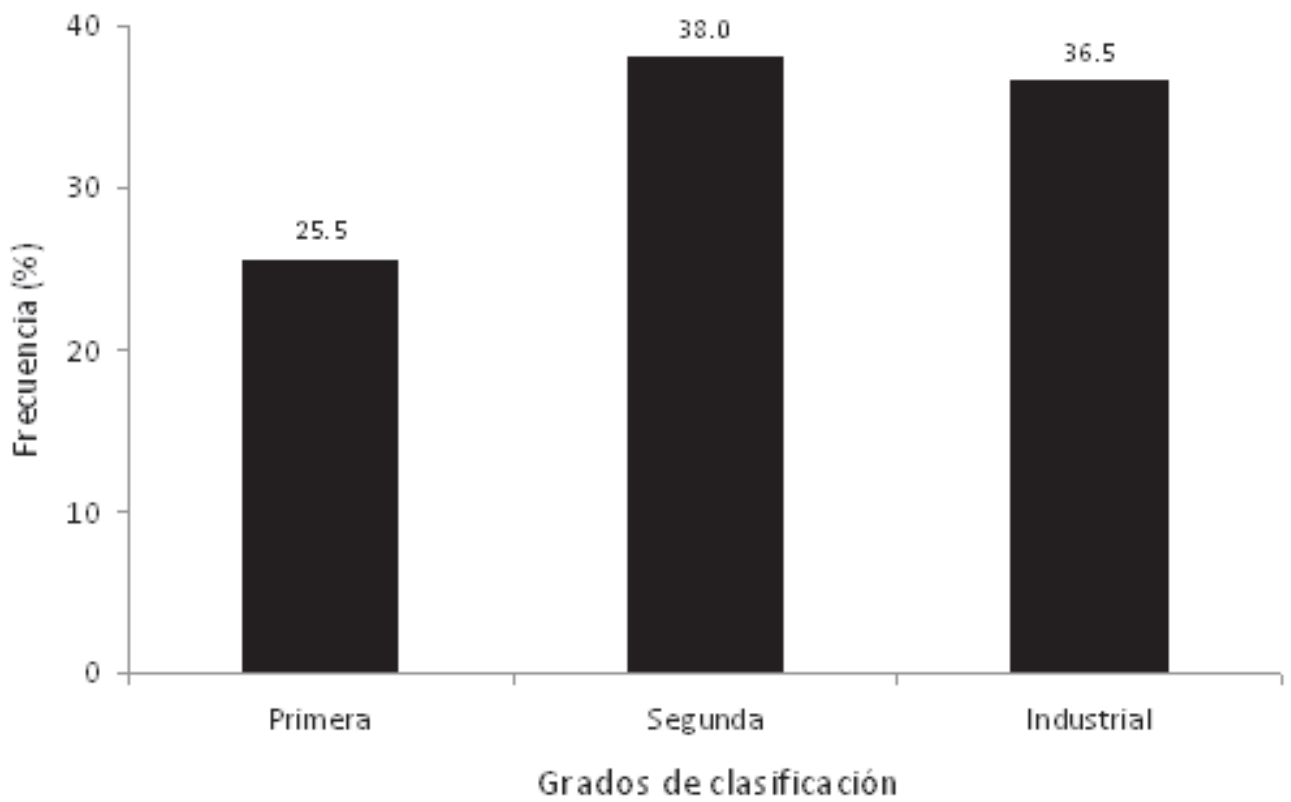

Figura 1. Canales de alpacas adultas sacrificadas en el camal de Huancavelica, Perú, según la clasificación de la carne

Las ecuaciones encontradas para la predicción del PCC y PCF en base al PV fueron: $\mathrm{PCC}=(4.190+0.464) \times \mathrm{PV}$ y $\mathrm{PCF}=$ $(3.933+0.461) \times$ PV. Los coeficientes de regresión en el análisis de varianza fueron altamente significativos $(\mathrm{p}<0.01)$, siendo los coeficientes de determinación de $68.5 \mathrm{y}$ $68.3 \%$, respectivamente.
En el Cuadro 3 se muestran los efectos del grado de clasificación de la carne sobre el PV, PCC, PCF y rendimiento de las carcasas de las alpacas beneficiadas, donde se observa que existe una diferencia altamente significativa sólo con respecto a estas tres primeras variables independientes en estudio. Se obtuvo menores pesos para canales de 
Cuadro 2. Correlaciones entre peso vivo ( $\mathrm{PV}$ ), peso de canal caliente (PCC), peso de canal fría(PCF) y rendimiento de carca sa en alpacas adultas sacrificadas en el camal de Huancavelica, Perú

\begin{tabular}{lccc}
\hline Variables & $\begin{array}{c}\text { Peso dela canal } \\
\text { fría }\end{array}$ & $\begin{array}{c}\text { Peso de la canal } \\
\text { caliente }\end{array}$ & Rendimiento \\
\hline Peso vivo & $.83^{* *}$ & $.83^{* *}$ & $-.24^{* *}$ \\
Peso de la canal fría & 1 & $.99^{* *}$ & $.35^{* *}$ \\
Peso de la canal caliente & $.99^{* *}$ & 1 & $.34^{* *}$ \\
Rendimi ento & $.35^{* *}$ & $.34 * *$ & 1 \\
\hline$* 0<0.01$ & & &
\end{tabular}

Cuadro 3. Efecto del grado de clasificación de la carne de alpaca sobre peso vivo (PV) y peso de la canal caliente (PCC) y fría (PCF), y rendimiento de la carcasa, en función a sexo y edad

\begin{tabular}{|c|c|c|c|c|c|c|c|c|c|c|}
\hline \multirow{2}{*}{ PV } & & \multirow[t]{2}{*}{$\mathrm{N}^{\circ}$} & \multicolumn{2}{|c|}{ Primera } & $\mathrm{N}^{\circ}$ & \multicolumn{2}{|c|}{ Segunda } & $\mathrm{N}^{\circ}$ & \multicolumn{2}{|c|}{ In dustrial } \\
\hline & & & $52.7^{\wedge}$ & & & $50.0^{b}$ & & & $54.7^{\star}$ & \\
\hline \multirow[t]{2}{*}{ Sexo } & Hembra & 21 & 58.1 & $54.7-615$ & 25 & 53.0 & $50.0-560$ & 39 & 566 & $54.4-58.9$ \\
\hline & & 30 & 490 & & 51 & 7.7 & & 34 & 52.5 & \\
\hline E dad & $\begin{array}{l}4 \mathrm{~d}^{\mathrm{d}} \\
\text { B. } 11 \mathrm{ena}^{2}\end{array}$ & $\begin{array}{l}40 \\
11\end{array}$ & $\begin{array}{l}51.8 \\
56.3\end{array}$ & & $\begin{array}{l}43 \\
33\end{array}$ & $\begin{array}{l}48.4 \\
50.8\end{array}$ & & $\begin{array}{l}35 \\
38\end{array}$ & $\begin{array}{l}552 \\
543\end{array}$ & \\
\hline \multirow{3}{*}{$\begin{array}{l}\text { PCC } \\
\text { Sexo }\end{array}$} & & & $29.0^{n}$ & & & $26.9^{b}$ & & & $29.7^{\mathrm{A}}$ & \\
\hline & & 21 & 31. & & 25 & 27.8 & & & & \\
\hline & Mach & 30 & & & & & & & & \\
\hline \multirow[t]{2}{*}{$E$ dad } & $4 d$ & 40 & 28.5 & & 43 & 26.4 & & & & \\
\hline & B. 11 ena & 11 & 30.6 & & 33 & 27.5 & & 38 & 295 & \\
\hline \multirow{3}{*}{$\begin{array}{l}\text { PCF } \\
\text { Sexo }\end{array}$} & & & $28.5^{n}$ & & & $26.5^{\mathrm{b}}$ & & & $29.3^{*}$ & \\
\hline & onpro & 21 & 30.8 & & 25 & 27.4 & & 39 & & \\
\hline & & & & & & & & & & \\
\hline \multirow[t]{2}{*}{$E$ dad } & $4 \mathrm{~d}$ & 40 & 28. & 26.3 & 43 & 26. & & 35 & 295 & \\
\hline & B. llena & 11 & 30.2 & $26.6-337$ & 33 & 27.1 & $25.7-285$ & 38 & 290 & $27.5-30.6$ \\
\hline \multicolumn{2}{|c|}{ Rendimiento } & & $54.2^{2}$ & & & $53.7^{2}$ & & & $53.7^{2}$ & \\
\hline \multirow[t]{2}{*}{ Sexo } & & 21 & & & 25 & & & 39 & 537 & \\
\hline & & & 55.1 & & & & & 3 & & \\
\hline \multirow[t]{2}{*}{$E$ dad } & & 40 & 54.4 & & 4 & 53 & & 3 & 53 & \\
\hline & & & 53.9 & $48.4-593$ & 33 & & $51.7-556$ & 38 & 538 & $52.1-55.4$ \\
\hline
\end{tabular}

14 diertes: ${ }^{2}$ Boca llena

ab Superíndices diferentes para Peso Vivo (PV), Peso de Canal Caliente ( $\mathrm{PCC}$ ), Peso de Canal Fría (PCF) y Rendimiento respecto al grado de calificación indican diferencias estadísticas al nivel de $p<0.05$ 
Segunda en comparación con canales con grados Primera e Industrial $(\mathrm{p}<0.05)$. Los rendimientos de las carcasas no se vieron afectados por el grado de clasificación.

\section{Discusión}

Las medias de PV, PCC y PCF obtenidas en el presente estudio fueron superiores al estudio de Cristofanelli et al. (2005) en alpacas machos de dos años, debido posiblemente a una mejor condición corporal producto de una mejor oferta del forraje, así como también al efecto edad, toda vez que el peso se incrementa hasta los 6 años (Castellaro et al., 1998). Por otro lado, los pesos de las alpacas fueron obviamente inferiores a los pesos reportados en llamas, considerándose las diferencias existentes en tamaño entre especies (Cristofanelli et al., 2004, 2005).

El efecto del sexo sobre PV, PCC y PCF se debería a que gran parte de los machos beneficiados correspondían a animales de descarte y generalmente de condición corporal baja, debido a que son usualmente relegados a pasturas de inferior calidad y cantidad; mientras que las hembras fueron mayormente animales en gestación que habían sido alimentadas en base a mejores pasturas por su condición fisiológica y que, sin embargo, debido a diversas razones (oportunidad de venta y necesidad de recursos económi$\cos$, entre otros), fueron beneficiadas para carne. Asimismo, el PCC y PCF, al estar altamente correlacionados con el PV, era de esperarse que también fueran superiores a lo reportado en otras situaciones. Debe indicarse que las hembras en gestación no sólo dirigen recursos nutritivos al feto, sino también a su propio organismo con una mayor eficiencia en la utilización de los alimentos, con el fin de incrementar significativamente sus reservas corporales para afrontar la futura lactación (Lawrence y Fowler, 2002).

El rendimiento de la carcasa varió entre 52.0 a $55.1 \%$, sin que se haya encontrado diferencia estadística por efecto del grado de clasificación, sexo o edad. Los rendimientos hallados se encuentran por encima a los encontrados por Ampuero y Alarcón (1989), aunque concordantes con Tellez $(1988,1996)$, Bustinza et al. (1975) y Mateo et al. (2010). La evidente diferencia de peso entre el PCC y PCF como consecuencia de las 12 horas de oreo, fue de 423 y $422 \mathrm{~g}$ para hembras y machos, respectivamente, y de 435 y $407 \mathrm{~g}$ para animales de 4 dientes y boca llena, respectivamente, aunque sin diferencia estadística por efecto de la edad, sexo o grado de clasificación. Se espera que las mermas sean mayores cuando se sacrifican animales más jóvenes (menores de dos años), pues poseen carnes más tiernas y con mayor contenido de agua (Polidori et al., 2007); sin embargo, en el presente trabajo sólo se tuvo animales mayores de tres años.

El grado de clasificación cárnica estuvo afectado por la edad y el peso corporal, concordante con otros estudios (Téllez, 1988). Asimismo, se debe considerar que de acuerdo a la Norma Técnica Peruana 201.043 (INDECOPI, 2005), canal Extra corresponde al proveniente de machos castrados con hasta cuatro dientes permanentes, con muy buen acabado, conformación, evidente grasa de cobertura e infiltración; canal Primera corresponde a machos castrados y hembras con hasta seis dientes permanentes con buen acabado de conformación; mientras que carcasas de machos y hembras con regular acabado y conformación son considerados como de Segunda, y que carcasas de animales, sea la condición y edad respectiva, pero con presencia de parásitos o infecciones corresponden a canales industriales. En el presente trabajo, debido a que los animales beneficiados tenían más de 3 años (correspondiente a 4 dientes a más), y que ninguno era castrado, no se observó canales clasificados como Extra.

La explicación de la asociación significativa del grado de clasificación de la carcasa con el PV, PCC y PCF se debería al hecho que el peso, conformación, cobertura de grasa, y la madurez del animal, así como la pre- 
sencia/ausencia de quistes parasitarios son influyentes en la determinación de la clasificación. Es importante mencionar, asimismo, que la infección por Sarcocystis aucheniae o sarcocistiosis, no solo atenta contra la salud del animal (La Perle et al., 1999), sino que continúa siendo un gran problema para la mayoría de grandes y pequeños productores de camélidos sudamericanos, afectando la producción y productividad, tanto por la pérdida de su valor comercial, el decomiso de la carcasa (Alva et al., 1980) y el rechazo de la carne en los mercados (Concha, 1999).

Las ecuaciones de predicción encontradas en base al peso vivo son buenas para predecir el PCC y PCF, pues tienen una alta correlación, concordante con los resultados de Faizur (2007) para el caso de cabras Black Bengal. Los resultados indican que por cada incremento en $1 \mathrm{~kg}$ de peso vivo se logra obtener $0.46 \mathrm{~kg}$, de canal o fría, lo cual es bastante cercano a lo encontrado en vacunos por Afolayan et al. (2002). Este resultado confirma la conveniencia de beneficiar animales de buen peso vivo a fin de obtener carcasas de buen peso, lo cual redundará en un buen retorno económico para el productor. Por lo tanto, el PV constituiría un buen criterio de selección con lo cual se lograría alcanzar no sólo objetivos de producción, sino también económicos (Cam et al., 2010).

\section{Conclusiones}

- Las medias de los pesos de animales en pie (peso vivo, PV), carcasas calientes (PCC) y carcasas frías (PCF) fueron mayores en hembras que en machos $(p<0.01)$ y en animales adultos que en animales jóvenes.

- Las carcasas obtenidas en el Camal de Huancavelica, proveniente del sacrificio de alpacas fueron en su mayor parte clasificadas como de segundo grado e industrial (38 y $36.5 \%$ respectivamente), no encontrándose canales de calidad extra.
- Se encontraron correlaciones positivas altamente significativas entre el peso vivo, peso de la carcasa caliente y peso de la carcasa fría.

- La predicción del peso de la canal caliente y el peso de la canal fría a partir del peso vivo se hace posible realizar a partir del peso vivo, pues el modelo tiene un alto coeficiente de determinación $(68 \%)$.

\section{LITERATURA CiTADA}

1. Afolayan RA, Deland MPB, Rutley DL, Bottema CDK, Ewers AL, Ponzoni RW, Pitchford WS. 2002. Prediction of carcass meat, fat and bone yield across diverse cattle genotypes using live-animal measurements. Anim Prod Aust 24: 13-16.

2. Alva J, Rojas M, Núñez A. 1980. Decomisos por parasitosis y su importancia económica en alpacas (Lama pacos). Rev Inv Pec, IVITA 5: 61-62.

3. Ampuero E, Alarcón V. 1989. Procesamiento de la carne de camélidos para charqui. Seminario-taller sobre producción, procesamiento, transformación y consumo de carne de camélidos domésticos. Puno, Perú: Proyecto Alpacas INIAA-COTESU.

4. Bustinza J, Ccopa A, Avila E, Bustinza V. 1975. Rendimiento de canal en alpacas tipo Huacaya por edades y sexo en el departamento de Puno. En: II Convención Internacional sobre Camélidos Sudamericanos. Juliaca, Perú.

5. Bustinza V, Medina G, Fernández E. 1985. Crecimiento de la alpaca. En: V Convención Internacional sobre Camélidos Sudamericanos. Cuzco, Perú.

6. Cam MA, Olfaz M y Soudan E. 2010. Body measurements reflect body weights and carcass yields in Karabayaka Sheep. Asian J Anim Vet Advan 5: 120-127. 
7. Castellaro G, García-Huidoro J, Salinas P. 1998. Alpaca liveweight variation and fiber production in Mediterranean range of Chile. J Range Manage 51: 509-513.

8. Concha S. 1999. Strategical plan of communication in marketing for the open consumption of alpaca meat in Arequipa - Peru. In: Progress in South American Camelids Research. Göttingen, Germany: The European Association for Animal Production. p 122-131.

9. Cristofanelli S, Antonini M, Torres D, Polidori P, Renieri C. 2004. Meat and carcass quality from Peruvian llama (Lama glama) and alpaca (Lama pacos). Meat Sci 66: 589-593.

10. Cristofanelli S, Antonini M, Torres D, Polidori P, Renieri C. 2005. Carcass characteristics of Peruvian llama (Lama glama) and alpaca (Lama pacos) reared in the Andean Highlands. Small Rumin Res 58: 219-222.

11. Fairfield T. 2006. The politics of livestock sector policy and the rural poor in Peru. Pro-Poor Livestock Policy Initiative, Food and Agriculture Organization. PPLPI Working Paper $\mathrm{N}^{\circ}$ 32. Rome: FAP. 70 p.

12. Faizur MD. 2007. Prediction of carcass weight from the body characteristics of Black Bengal goats. Internat J Agric Biol 3: 431-434.

13. Flores JA. 1982. Causas que originaron la actual distribución espacial de las alpacas y llamas. En: Miliones L, Tomoedea H (eds). El hombre y su ambiente en los Andes centrales. Senri Ethnological Studies 10: 63-92.

14. Hack W. The Peruvian alpaca meat and hide industries. Rural Industries Research \& Development Corporation. Publication No. 01/19. Barton, Australia: RIRDC. $11 \mathrm{p}$.

15. INDECOPI. 2005. Norma Técnica Peruana 201.043. Carne y productos cárnicos. Definiciones, requisitos y clasificación de las carcasas y carne de alpacas y llamas. CTN 008: Carne y Productos Cárnicos. R. 47-2005-CRTINDECOPI. $13 \mathrm{p}$.
16. La Perle K, Silverio F, Anderson D, Blomme A. 1999. Dalmeny disease in an alpaca (Lama pacos): Sarcocystiosis, eosinophilia myositis and abortion. J Comp Path 121: 287-293.

17. Lawrence TLJ, Fowler VR. 2002. Growth in farm animals. $2^{\text {nd }}$ ed. UK: CAB Publishing. $347 \mathrm{p}$.

18. Mamani G. 1989. La crianza de camélidos sudamericanos domésticos en comunidades alpaqueras y la producción de carne. Seminario-Taller sobre producción, procesamiento, transformación y consumo de carne de camélidos domésticos. Puno, Perú: Proyecto Alpacas INIAA-COTESU.

19. Mateo J, Salvá B, Ramos D., Caro I, Prieto B, Gonzáles A. 2010. Características de la carne de alpaca y procesamiento de charqui en los departamentos de Puno y Cusco (Perú). León, España: Celarayn. $64 \mathrm{p}$.

20. Pérez P, Maino M, Guzmán R, Vaquero A, Köbrich C, Pokniak J. 1999. Carcass characteristics of llamas (Lama glama) reared in Central Chile. Small Rumin Res 37: 93-97.

21. Polidori P, Renieri C, Antonini M, Passamonti P, Pucciarelli F. 2007. Meat fatty acid composition of llama (Lama glama) reared in the Andean highlands. Meat Sci 75: 366-368.

22. Saadoun A, Cabrera MC. 2008. A review of the nutritional content and technological parameters of indigenous sources of meat in South America. Meat Sci 80: 570-581.

23. Salvá BK, Zumalacárregui JM, Figueira AC, Osorio MT, Mateo J. 2009. Nutrient composition and technological quality of meat from alpacas reared in Peru. Meat Sci 82: 450455.

24. UNECE. 2006. Llama/Alpaca meat, carcasses and cuts. Agricultural Standards Unit, United Nations Economic Commission for Europe. Geneve: United Nation Publications. 36 p.

25. RTC. 1995. Reglamento tecnológico de carnes. DS 22-95-AG. [Internet], [31 octubre 2011]. Disponible en: http:// 
www.senasa.gob.pe/RepositorioAPS/0/ 0/JER/SEC_NOR_INS_2/DS\%200221995\%20(2).pdf

26. Téllez JG. 1988. Procesamiento de carnes de alpaca. Seminario Taller sobre producción, transformación, procesamiento y consumo de carnes de camélidos sudamericanos. Puno, Perú: Alpacas INIAA-COTESU.

27. Wheeler J. 1993. South American camelids: past, present and future. In: Proc $1^{\text {st }}$ European Symposium on South American camelids. Bonn, Germany. p 13-28. 